Elsevier required licence: (C) <2017>. This manuscript version is made available under the CC-BY-NC-ND 4.0 license http://creativecommons.org/licenses/by-nc-nd/4.0/ 


\title{
pIP40a, a type 1 IncC plasmid from 1969 carries the integrative element and a novel class II mercury resistance transposon
}

\author{
Christopher J. Harmer ${ }^{\star}$, Mohammad Hamidian, Ruth M. Hall \\ School of Life and Environmental Sciences, The University of Sydney, NSW 2006, Australia
}

\section{A R T I C L E I N F O}

\section{Keywords:}

IncC

GIsul2

Genomic island

Antibiotic resistance

Transposon

\begin{abstract}
A B S T R A C T
The $167.5 \mathrm{~kb}$ sequence of the conjugative IncC plasmid pIP40a, isolated from a Pseudomonas aeruginosa in 1969, was analysed. pIP40a confers resistance to kanamycin, neomycin, ampicillin, sulphonamides and mercuric ions, and several insertions in a type 1 IncC backbone were found, including copies of IS3, Tn1000 and a novel mercury resistance transposon, Tn6182. The antibiotic resistance genes were in two locations. Tn6023, containing the aphA1 kanamycin and neomycin resistance gene, is in a partial copy of $\operatorname{Tn} 1 / \operatorname{Tn} 2 / \operatorname{Tn} 3$ (bla TEM, ampicillin resistance) in the $k f r A$ gene, and the sul2 sulphonamide resistance gene is in the integrative element GIsul2 in the position of ARI-B islands. The $11.5 \mathrm{~kb}$ class II transposon Tn6182 is only distantly related to other class II transposons, with at most 33\% identity between the TnpA of Tn6182 and TnpA of other group members. In addition, the inverted repeats are $37 \mathrm{bp}$ rather than $38 \mathrm{bp}$, and the likely resolution enzyme is a tyrosine recombinase (TnpI). Re-annotation of GIsul2 revealed genes predicted to confer resistance to arsenate and arsenite, but resistance was not detected. The location of GIsul2 confirms it as the progenitor of the ARI-B configurations seen in many IncC plasmids isolated more recently. However, GIsul2 has integrated at the same site in type 1 and type 2 IncC plasmids, indicating that it targets this site. Analysis of the distribution of GIsul2 revealed that it in addition to its chromosomal integration site at the $3^{\prime}$-end of the guaA gene, it has also integrated into other plasmids, increasing its mobility.
\end{abstract}

\section{Introduction}

IncC plasmids have long been known to play an important role in the dissemination of antibiotic and mercuric ion resistance determinants. They were first recovered in Paris in the late 1960s (Chabbert et al., 1972; Witchitz and Chabbert, 1971). However, it was not until 2007 that an IncC plasmid was sequenced (Welch et al., 2007). Plasmid pIP40a was originally recovered from a clinical Pseudomonas aeruginosa isolate in Paris in 1969 and classified as IncP-3. It was transferred by conjugation into an Escherichia coli host and was assigned to incompatibility group IncC using R55, also recovered in 1969 in Paris (Witchitz and Chabbert, 1971), as the IncC reference plasmid (Chabbert et al., 1972). For a period of time, IncC plasmids were combined with the IncA plasmids as IncA/C (Datta and Hedges, 1972). Later, they were split into $A / C_{1}$ and $A / C_{2}$ (Carattoli et al., 2006), corresponding to the Inc $A$ and IncC groups respectively (Harmer et al., 2016b). However, as Inc A and IncC plasmids were known to be compatible (Datta and Hedges, 1973), the designation IncA/C is incorrect and they have been separated again into IncA, of which RA1 is the only sequenced example, and the more common and more important IncC group (Harmer and

\section{Hall, 2015).}

A comparison of the 29 Inc $C$ plasmid sequences available in the public domain in 2014 revealed that there were two distinct lineages, type 1 and type 2 (Harmer and Hall, 2014). The backbones of IncC plasmids from the two lineages differ by $\sim 1 \%$ and are also distinguished by two regions where alternate sequences are found, orf1832 in type 1 or orf1847 in type 2 , and $r h s 1$ in type 1 or $r h s 2$ in type 2 , and two small additional segments (i1 and i2) in the type 2 plasmid backbone (Harmer and Hall, 2014). It is clear that the type 1 and type 2 IncC plasmids have separate evolutionary histories, and we recently developed a PCR typing scheme to distinguish type 1 and type 2 plasmids (Harmer and Hall, 2016). However, sequences provide more detailed information.

One of the key stages in the evolution of type 1 and type 2 IncC plasmids has been the acquisition of antibiotic resistance islands, and sub-lineages can be identified based on the location of resistance islands, which can vary internally. The majority of recently isolated type 1 plasmids have the ARI-A island, and are likely to be descended from a common ancestor (Harmer and Hall, 2015). However, surprisingly the ARI-B antibiotic resistance island, containing sul2 and all or part of the 
small mobile element CR2, is found in the same location in both type 1 and type 2 IncC plasmids (Harmer and Hall, 2015; Harmer and Hall, 2014). A variety of ARI-B configurations carrying additional antibiotic resistance genes have been observed (see Table 5 and Fig. 7 in (Harmer and Hall, 2015)). Recently, it has been shown that the sul2 gene is part of a $15 \mathrm{~kb}$ integrative element GIsul2 (Nigro and Hall, 2011; Hamidian and Hall, 2017). In three sequenced IncC plasmids (R55, pEA1509 and pCFSAN001921), ARI-B is bounded by the two outer ends of GIsul2 (Harmer and Hall, 2015; Nigro and Hall, 2011), but the central portion of the genomic island has been lost. Hence, ARI-B appears to have originally formed via the integration of GIsul2. However, no IncC plasmid carrying a complete copy of GIsul2 had been seen until recently. Whether GIsul2 was acquired independently by each lineage, or whether recombination between the two lineages has occurred remains to be resolved.

R55, the oldest IncC plasmid known to be type 2 (Harmer and Hall, 2014), has been sequenced and it had already acquired a complex antibiotic and mercury resistance region (Doublet et al., 2012). However, the type 1 plasmids are the most important, as members of this group have acquired the bla $a_{\mathrm{CMY}}$ cephalosporin resistance gene and more recently the $b l a_{\mathrm{NDM}}$ carbapenem resistance gene which has been acquired by the ARI-A resistance island. Among the type 1 plasmids, pDGO100, which was recovered in 1981 (Groot Obbink et al., 1985), was one of the oldest that had been sequenced so far (Harmer et al., 2016b). pDGO100 had acquired the ARI-A resistance island but lacks an ARI-B island. The next oldest, p199061_160, wasn't recovered until 1991 (Fernandez-Alarcon et al., 2011). Though over 100 IncC plasmids have been sequenced since 2007 (last searched 1st February 2017), they mostly originate from the contemporary period and many are identical to one another or very closely related (Harmer and Hall, 2015). Hence, they do not add a great deal to our understanding of the longer-term evolutionary history of the type 1 IncC plasmids, or their role in the emergence of antibiotic and heavy metal resistance.

Recently, we showed that pIP40a is type 1 (Harmer and Hall, 2016). Hence, both type 1 and type 2 IncC plasmids were circulating in Paris in the late 1960s and contributing to the development of antibiotic resistance. We also used PCR to show that ARI-B in pIP40a contains both ends of GIsul2, and confirmed by sequencing (GenBank accession number KX709966) that it contains a complete copy of GIsul2 (Harmer and Hall, 2016). Here, we present a detailed analysis of the complete sequence of pIP40a, aimed at increasing our understanding of the evolution of IncC plasmids and of the role of genomic island GIsul2 in spreading the sul2 sulphonamide resistance gene. A novel class II transposon was also identified.

\section{Methods}

\section{1. $p I P 40 a$}

Plasmid 40a was originally recovered in 1969 in Paris, France, from a $P$. aeruginosa clinical isolate that had caused a urinary tract infection (Chabbert et al., 1972) and later assigned an Institute Pasteur (IP) number. pIP40a was recorded as conferring resistance to kanamycin, neomycin, ampicillin, sulphonamides, and mercuric ions. An E. coli containing pIP40a was obtained from the UK National Type Culture Collection via Professor David Gordon (Australian National University).

To determine the frequency of transfer, pIP40a was transferred overnight $(16 \mathrm{~h})$ on solid media from nalidixic acid resistant $\left(\mathrm{Nx}^{\mathrm{R}}\right)$ E. coli DH5- $\alpha$ to streptomycin resistant $\left(\mathrm{Sm}^{\mathrm{R}}\right)$ E. coli UB1637 as described previously (Harmer et al., 2016b).

\subsection{Antibiotic and heavy metal susceptibility testing}

Antibiotic susceptibility profiles were determined as described previously (Bailey et al., 2010). Resistance to mercuric ions was tested by growing strains overnight at $37^{\circ} \mathrm{C}$ on LB agar supplemented with
$25 \mu \mathrm{g} / \mathrm{ml} \mathrm{HgCl}_{2}$. Resistance to arsenate and arsenite was tested by growth on $\mathrm{LB}$ agar containing $5 \mathrm{mM} \mathrm{NaAsO}_{2}$ or $20 \mathrm{mM} \mathrm{Na}_{2} \mathrm{HAsO}_{4}$, respectively.

\subsection{Analysis of PIP40a sequence}

The complete sequence of pIP40a assembled previously (Harmer and Hall, 2016), and submitted to GenBank under accession number KX709966, was analysed. Here, additional sequence data that increased the read depth from 12 to 29 -fold was assembled and no differences were found. The copy number of pIP40a was assessed using contig coverage relative to that of seven chromosomal genes. Reading frames of $>300 \mathrm{bp}$ (100 aa) were predicted using Gene Construction Kit (Textco BioSoftware, Raleigh, NC, USA) and were annotated using the annotations of the IncC plasmids pRMH760 (GenBank accession number KF976462) and pR148 (GenBank accession number CP000603) as a guide. Insertion sequences were identified using IS Finder (https:/ / www-is.biotoul.fr) (Siguier et al., 2006), and antibiotic resistance genes were identified using ResFinder 2.1 (https://cge.cbs.dtu.dk/services/ ResFinder/). Pfam (http://pfam.xfam.org/) was used to assist in the prediction of the function of proteins encoded by genes whose function has not been experimentally determined.

The backbone of pIP40a was derived by removing the transposons, insertion sequences and antibiotic resistance islands, and any duplication created when they were inserted. The backbone was compared to other complete IncC plasmids present in the Genbank non-redundant database. To facilitate comparisons, the backbones of these plasmids were generated in the same manner by removing all insertions, then circularising the sequences and reopening them at the same location, namely 1139 bp upstream of repA.

\subsection{Detection of excised GIsul2}

To test for excision of GIsul2 from pIP40a, E. coli UB1637 $\left(\mathrm{Sm}^{\mathrm{R}}\right)$ containing pIP40a was grown overnight in Luria Broth without selection. Whole cell genomic DNA was extracted and used as the template in a PCR with primers RH1879 (5'-ATCGTGCCAAACACAAACAA-3') and R3R (5'-GAATGCATAACGACGAGTTTGG-3'). These primers have been utilised previously in different combinations (Harmer and Hall, 2016), to detect the excised circular product. PCR conditions were as published previously (Harmer and Hall, 2016). To test for low frequency excision, $1 \mu \mathrm{l}$ of the PCR reaction was used as a template for a second round of amplification using the same primers.

\section{Results}

\subsection{Plasmid pIP40a}

pIP40a was previously reported to contain genes conferring resistance to ampicillin, kanamycin and sulphonamides (Chabbert et al., 1972). E. coli containing pIP40a were also able to grow on plates containing $25 \mu \mathrm{g} / \mathrm{ml} \mathrm{HgCl}$, indicating the presence of a mer module. pIP40a had a unit copy number. The efficiency of transfer of pIP40a ( $E$. coli $\mathrm{DH} 5-\alpha$ to E. coli $\mathrm{UB} 1637)$ was $4.38 \times 10^{-5} \mathrm{Ap}^{\mathrm{R}} \mathrm{Km}^{\mathrm{R}} \mathrm{Su}^{\mathrm{R}}$ transconjugants per donor (average of three independent determinantions). This is between 100- and 1000-fold lower than the conjugation frequencies of other well-studied type 1 IncC plasmids such as pRMH760 $\left(6.4 \times 10^{-2}\right.$ (Harmer and Hall, 2014)), pDGO100 $\left(3 \times 10^{-3}\right.$ (Harmer et al., 2016b)) or the type 2 pVCR94 $\Delta \mathrm{X}\left(3.5 \times 10^{-3}-1 \times 10^{-2}\right.$ (Carraro et al., 2014b, Carraro et al., 2014a)).

The pIP40a sequence (GenBank accession number KX709966) was $167,554 \mathrm{bp}$, and the sequence of the backbone confirmed recent findings (Harmer and Hall, 2016) that pIP40a is a type 1 IncC plasmid. Several insertions were identified in the backbone of pIP40a, and their locations are shown in Fig. 1. An IS3, flanked by a $3 \mathrm{bp}$ target site duplication (TSD), has interrupted the rhs1 gene as noted previously 

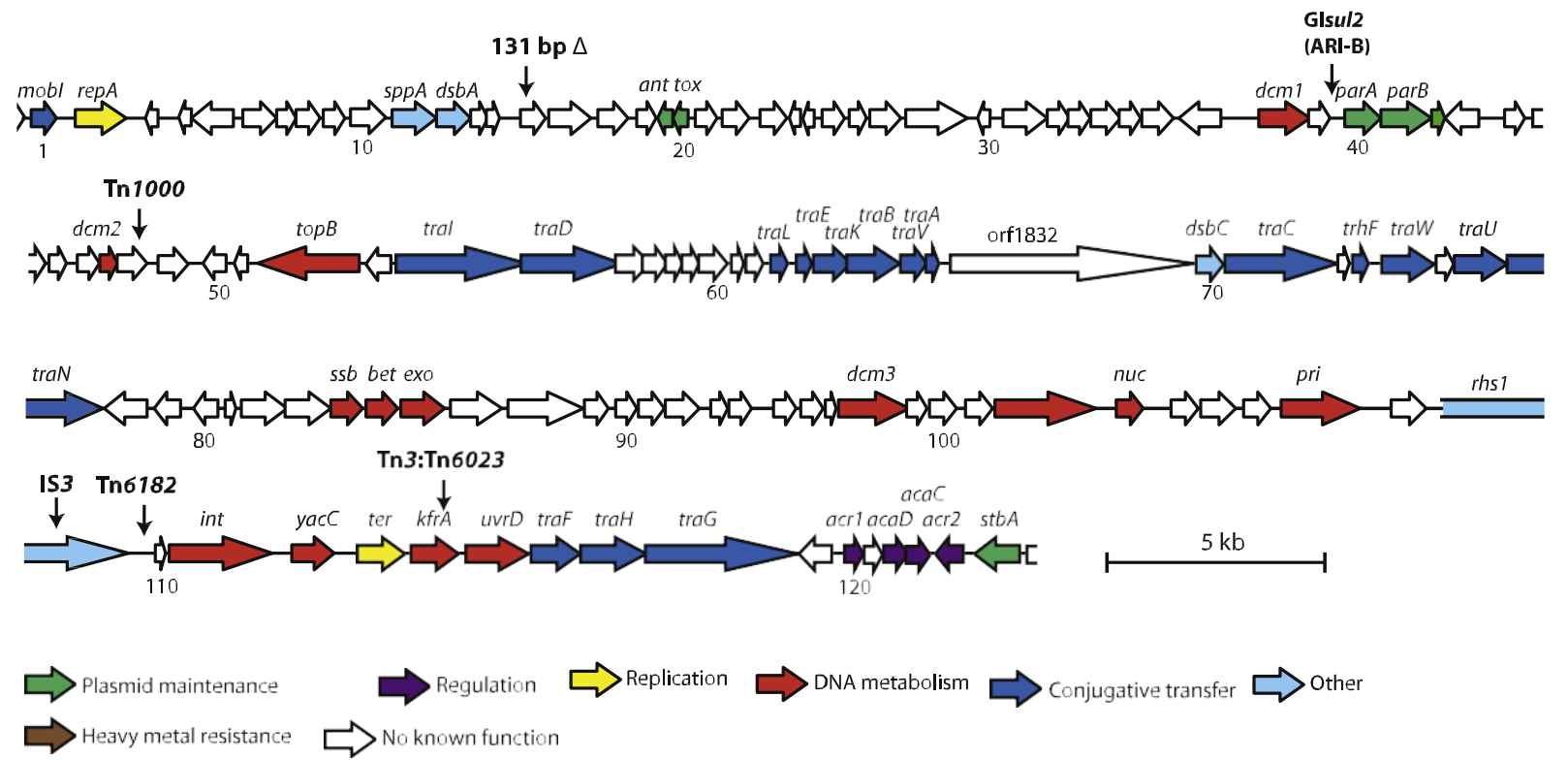

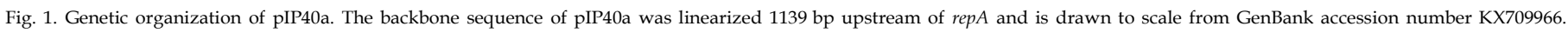

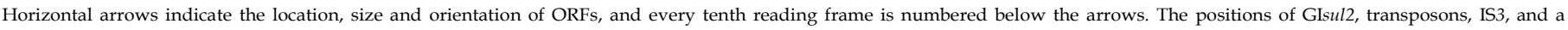

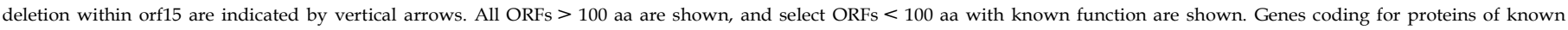

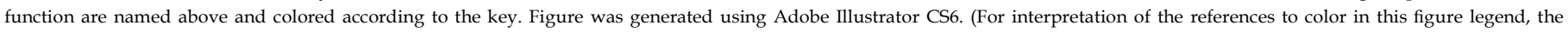
reader is referred to the web version of this article.)

(Harmer and Hall, 2016). pIP40a also includes a partial copy of Tn1/ $\mathrm{Tn} 2 / \mathrm{Tn} 3$ interrupted by the kanamycin resistance transposon Tn6023 (Cain and Hall, 2011), which includes the aphA1b gene conferring resistance to kanamycin and neomycin $\left(\mathrm{Km}^{\mathrm{R}} \mathrm{Nm}^{\mathrm{R}}\right)$, a complete copy of the class II transposon Tn1000 (also known as gamma delta)(Broom et al., 1995), and a novel mercury-resistance transposon which we named Tn6182. pIP40a includes GIsul2, containing sul2 $\left(\mathrm{Su}^{\mathrm{R}}\right)$, as the ARI-B resistance island. Whilst most known type 1 IncC plasmids carry an ARIA resistance region (Harmer and Hall, 2015), pIP40a does not, and the plasmid backbone is continuous at the ARI-A location. Hence pIP40a, only the second sequenced type $1 \mathrm{IncC}$ plasmid that does not contain an ARI-A island (Harmer and Hall, 2015), belongs to a type 1 lineage that predates the entry of the ancestral form of ARI-A.

\subsection{The backbone of $p I P 40$}

Removal of ARI-B, the transposons, the IS and the duplications they created, generated a plasmid backbone of 127,670 bp (Fig. 1) that includes a replication initiation gene $(\operatorname{rep} A)$, genes for conjugative transfer (tra), plasmid partitioning (par), a variety of other genes with predicted functions, and open reading frames with no known function that have been described elsewhere (Harmer and Hall, 2015; Hancock et al., 2017). The pIP40a backbone is $133 \mathrm{bp}$ smaller than the typical type 1 IncC backbone of 127,803 bp (Harmer and Hall, 2014), due to a $131 \mathrm{bp}$ deletion internal to an open reading frame with no known function (see Fig. 1), and the absence of two single base-pairs in intergenic regions of the backbone, that are present in all other type 1 IncC plasmids. A BLAST search using the pIP40a backbone as a query showed that it is most closely related to the backbone of pRMH760 (GenBank accession number KF976462). In addition to the $131 \mathrm{bp}$ deletion in pIP40a and a 2358 bp deletion in the rhs1 gene of pRMH760, the two backbones differ by a total of 63 single nucleotide polymorphisms (SNPs) distributed throughout the backbone. The majority of the SNPs are intergenic (21/63) or in ORFs of no known function $(28 / 63)$. Seventeen are in genes of known function, of which nine are silent substitutions. The remaining eight SNPs result in single amino acid changes in TraI, TraL, TraC, TraN, Int, and Yac, and two substitutions in Dcm3. pR148 (GenBank accession number CP000603), which includes the $2358 \mathrm{bp}$ missing from pRMH760, is the next closest relative to pIP40a, differing by 65 SNPs, most shared by pRMH760. The non-synonymous substitutions in the tra genes could be responsible for the low transfer frequency.

\section{3. $\operatorname{Tn} 1 / \operatorname{Tn} 2 / \operatorname{Tn} 3:: \operatorname{Tn} 6023$}

The $b l a_{\mathrm{TEM}-1}$ ampicillin resistance gene is found in a remnant of a $\operatorname{Tn} 1 / \operatorname{Tn} 2 / \operatorname{Tn} 3$ transposon (Fig. 2a) that has inserted into the $k f r A$ gene of the pIP40a backbone (Fig. 1) and is flanked by a $5 \mathrm{bp}$ TSD. The $2641 \mathrm{bp}$ aphA1b-containing IS26-bounded transposon Tn6023 (GenBank accession number GU562437) has subsequently inserted in this Tn (Fig. 2a), and an IS26-mediated deletion arising from either one or both of the IS26 bounding Tn6023 has removed $1935 \mathrm{bp}$ of the Tn, removing res and truncating the $\operatorname{tn} A$ gene. As this region includes the features that distinguish Tn1, Tn2 and Tn3 from each other (Partridge and Hall, 2005; Bailey et al., 2011), it is not possible to identify the specific variant. Tn6023 is not flanked by a TSD due to this deletion.

Tn6023 is unusual because the two IS26 flanking aphA1b are inversely oriented, whereas in most IS26-bounded transposons the IS are in the same orientation. Tn6023 was first described as a component of a larger transposon (GenBank accession number GU562437) in an IncHI2 plasmid, pSRC125, recovered from a $S$. enterica of bovine origin in Australia in the year 2000 (Cain and Hall, 2011). However, the presence of Tn6023 in pIP40a shows clearly that it arose at least three decades earlier. In pSRC125, Tn6023 is within Tn5393 and is flanked by an 8 bp TSD. A BLAST search of the GenBank nucleotide database using Tn6023 as a query revealed that it has since been found in the chromosomes or plasmids of a number of bacterial species, including Corynebacterium singular, Pseudomonas putida, P. aeruginosa, Serratia marcescens, Acinetobacter baumannii and Proteus mirabilis. In most of these sequences, Tn6023 is not flanked by a TSD. However, in five Corynebacterium genome sequences (GenBank accession numbers CP010827, CP009211, AM942444, FN825254 and CP004085), Tn6023 is in the same position in Tn5393 as in pSRC125 flanked by the same 8 bp duplication.

\subsection{Tn6182, a new class II mercury resistance transposon}

A novel 11,514 bp transposon, Tn6182 (Fig. 2b), is inserted in the 
a

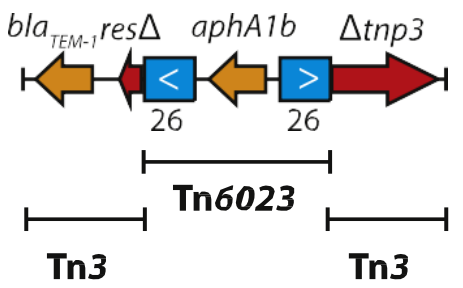

b
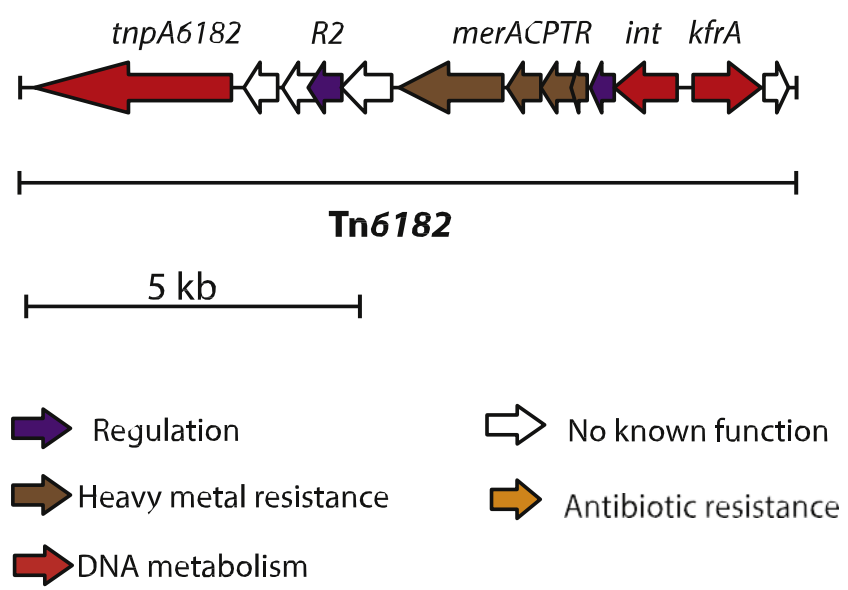

Fig. 2. Resistance transposons in pIP40a. a) Tn3::Tn6023. b) Novel mercury resistance transposon Tn6182. The transposons are drawn to scale from GenBank accession no. KX709966, and are drawn in the orientation consistent with Fig. 1. IS26 are shown as open boxes with $>$ or $<$ inside indicating the orientation. Genes coding for proteins of known function are named above and colored according to the key. Figure was generated using Adobe Illustrator CS6. (For interpretation of the references to color in this figure legend, the reader is referred to the web version of this article.)

intergenic region downstream of rhs1 in pIP40a (Fig. 1). Tn6182 is bounded by $37 \mathrm{bp}$ terminal inverted repeats and is flanked by a $5 \mathrm{bp}$ TSD. Annotation of all open reading frames encoding proteins $>50$ amino acids (see Table 1 and GenBank accession number KX709966 for full annotations), uncovered a gene encoding a 995 aa protein, identified by Pfam as a class II (Tn3 family) TnpA transposase (PF01526), at one end of Tn6182. TnpA6182 is co-linear with the unusually long transposases found in class II transposons, and phylogenetic analysis (Fig. 3a) with one or more representative transposases from each of the clades of the class II transposons defined recently (Nicolas et al., 2015) a

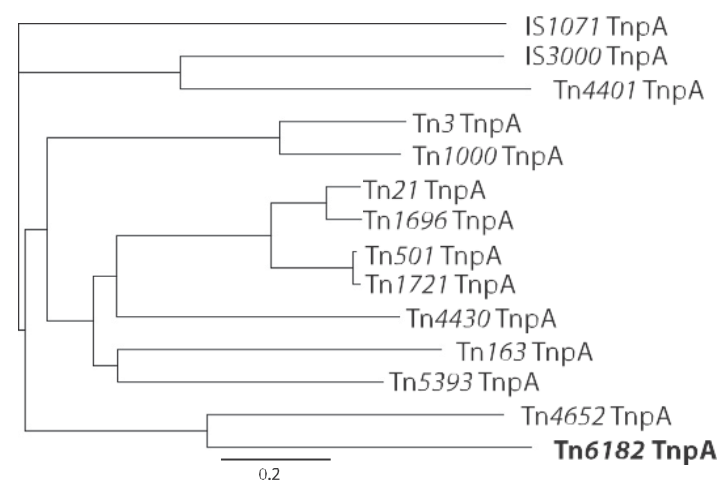

b

Tn21 IRL GGGGTCGTCTCAGAAAACGGAAAATAAAGCACGCTAAG
$\operatorname{Tn} 4652$ IRL GGGGTTATGCCGAGATAAGGCAAAAATTAGGACATTCGTTCTGTAA
$\operatorname{Tn} 6182$ IRL GAGGTCCGGCCGGTAAAAGCGTTGAATTTAGCCACTTC
$\operatorname{Tn} 6182$ IRR GAGGTCCGGCCGGTAAAAGCGTTGAATTTAGCCACTTT

Fig. 3. Comparison of features of Tn6182 and other class II transposons. a) Phylogenetic tree showing relatedness between the transposase proteins of selected class II transposons. The tree was generated using Geneious R7 using a neighbour-joining algorithm. b) Alignment of the left and right inverted repeats of Tn6182 with the inverted repeats of $\mathrm{Tn} 21$ and Tn4652. Bold letters indicate highly conserved G nucleotides. Vertical lines indicate shared bases.

showed that TnpA6182 is only distantly related to other class II transposases. The closest relative is the TnpA of Tn4652 (GenBank accession number X83686), with which it shares 33\% aa identity. The other class II transposases all share $<26 \%$ aa identity with the TnpA of Tn6182. Hence, Tn6182 likely represents a new clade.

The inverted repeats (IR) of Tn6182 also exhibit differences from those of other class II transposons. The four terminal bases (GGGG) of the $38 \mathrm{bp}$ IRs of all other class II transposons are completely conserved (see alignment in (Nicolas et al., 2015)). However, one of these bases is not conserved in the IRs of Tn6182 (GAGG) (Fig. 3b). The 38th base of the inverted repeats in all other class II transposons, a highly-conserved G nucleotide, is not present in Tn6182 (Fig. 3b). Hence, the IRs are one base pair shorter than the more usual $38 \mathrm{bp}$ IRs for other class II transposons. Many class II transposons include a tnpR gene encoding a

Table 1

Features of $\operatorname{Tn} 6182$

\begin{tabular}{|c|c|c|c|c|c|c|}
\hline Feature/gene & Position $^{\mathrm{a}}$ & Size (bp) & Product & Size $(\mathrm{aa})^{\mathrm{b}}$ & Pfam & Description \\
\hline IRL & $1-37$ & 37 & - & - & - & Inverted repeat \\
\hline $\operatorname{tnp} A 6182$ & $68-3052 c$ & 2985 & TnpA6182 & 995 & 01526 & Class II transposase \\
\hline orf & $3172-3735 c$ & 564 & Orf & 188 & - & Hypothetical protein \\
\hline orf & $3735-4127 c$ & 393 & Orf & 131 & - & Hypothetical protein \\
\hline$R 2$ & $4154-4684 c$ & 531 & $\mathrm{R} 2$ & 177 & 13411 & MerR family regulator \\
\hline orf & $4608-5405 c$ & 798 & Orf & 266 & 00563 & EAL domain family protein \\
\hline merA & $5420-7084 c$ & 1665 & MerA & 555 & 07992 & Mercuric reductase \\
\hline $\operatorname{merC}$ & $7125-7541 c$ & 417 & MerC & 139 & 03203 & Mercury resistance protein \\
\hline $\operatorname{mer} P$ & $7548-7823 c$ & 276 & MerP & 92 & 00403 & Mercury transport protein \\
\hline $\operatorname{mer} T$ & $7876-8223 c$ & 348 & MerT & 116 & 02411 & Mercury transport protein \\
\hline $\operatorname{mer} R$ & $8320-8709 c$ & 390 & MerR & 130 & 13411 & MerR family regulator \\
\hline $\operatorname{tnpI}$ & $8709-9728 c$ & 1020 & TnpI & 340 & 00589 & Tyrosine recombinase \\
\hline$k f r A$ & $9879-10,979$ & 1101 & KfrA & 367 & 11740 & Site-specific DNA binding protein \\
\hline orf & $11,102-11,431$ & 330 & Orf & 110 & - & Hypothetical protein \\
\hline IRR & $11,478-11,514$ & 37 & - & - & - & Inverted repeat \\
\hline
\end{tabular}

a Position 1 corresponds to position 131,414 of GenBank accession number KX709966. The letter "c" after the base range indicates the gene/orf is on the complimentary strand.

${ }^{\mathrm{b}}$ Length of product in amino acids (aa).

${ }^{\mathrm{c}}$ Open reading frame. 
Table 2

Amino acid identities of Mer proteins encoded in Tn6182 compared to Tn21, Tn501, and Tn1696.

\begin{tabular}{|c|c|c|c|c|c|c|}
\hline $\operatorname{Tn}$ & Accession no. & $\begin{array}{l}\text { MerA } \\
\% \text { (555aa) }\end{array}$ & $\begin{array}{l}\text { MerC } \\
\% \text { (139aa) }\end{array}$ & $\begin{array}{l}\text { MerP } \\
\% \text { (89aa) }\end{array}$ & $\begin{array}{l}\text { MerT } \\
\% \quad(116 a a)\end{array}$ & $\begin{array}{l}\text { MerR } \\
\%(130 a a)\end{array}$ \\
\hline $\operatorname{Tn} 6182$ & KX709966 & 100 & 100 & 100 & 100 & 100 \\
\hline $\operatorname{Tn} 21$ & AF071413 & $57(325 / 567)$ & $56(73 / 131)$ & $61(43 / 70)$ & $50(52 / 105)$ & $46(58 / 125)$ \\
\hline $\operatorname{Tn} 501$ & Z00027 & $57(323 / 564)$ & $-^{\mathrm{a}}$ & $64(45 / 70)$ & $46(51 / 111)$ & $47(59 / 125)$ \\
\hline Tn1696 & U12338 & $56(219 / 566)$ & $59(78 / 132)$ & $63(47 / 70)$ & $53(53 / 100)$ & $47(59 / 125)$ \\
\hline
\end{tabular}

${ }^{\text {a }}$ No MerC in Tn501.

TnpR serine recombinase (resolvase) that acts to resolve cointegrates, whilst in others, including Tn6182, a tnpI or int gene encoding a tyrosine recombinase (Pfam PF00589) replaces tnpR (Nicolas et al., 2015). Another gene, $k f r A$, encodes a DNA binding protein associated with plasmid replication (Pfam PF11740). Though there are similarly named genes in the IncC backbone that encode proteins sharing the same Pfam, the Tn6182 tnpI and $k f r A$ genes do not share any significant sequence similarity with those from IncC plasmids.

Like many other class II transposons, Tn6182 carries a mercuric ion resistance operon, and pIP40a confers resistance to mercuric ions. The mer module is novel, but the MerA, MerC, MerP, and MerT proteins share $46-64 \%$ amino acid identity with the corresponding Mer proteins in the well-characterised Tn21 (GenBank accession number AF071413), Tn501 (GenBank accession number Z00027) and Tn1696 (GenBank accession number U12338) mer operons (Table 2), allowing their functions to be deduced. However, unlike most other transposons that carry the mer operon at an outer end (Liebert et al., 1999; Partridge et al., 2001), the mer operon of Tn6182 (merACPTR) is located in the central portion of the transposon (Fig. 2b).

\subsection{GIsul2, the progenitor of ARI-B islands}

We recently proposed that the various ARI-B resistance islands seen in many type 1 and type 2 IncC plasmids arose from GIsul2 integrated into the IncC backbone (Harmer and Hall, 2015; Harmer and Hall, 2016). The pIP40a sequence confirmed this as the ARI-B island of pIP40a is a complete copy of the 15,460 bp integrative element GIsul2 (Fig. 4a). Re-examination of all GIsul2 open reading frames predicting proteins longer than 50 amino acids (see Table 3 and GenBank accession number KX709966 for full annotations) has enhanced the earlier annotations of GIsul2 (Nigro and Hall, 2011). In addition to the previously described int, repA, repC, trbJ, trbL, resG, $r c r 2$, and sul2 genes (Nigro and Hall, 2011), a number of genes that had been overlooked were identified.

GIsul2 includes an apparently complete arsenate/arsenite resistance operon ( $\operatorname{ars} B C H R$, Fig. 4 a) that encodes proteins that are distantly related to those from other previously characterised ars operons such as those found in S. enterica R46 (GenBank accession number NC003292), E. coli R773 (GenBank accession number J02591), and P. aeruginosa PAO1 (GenBank accession number NC002516). The module in pIP40a encodes ArsC and ArsB homologues that are over $40 \%$ identical to those of P. aeruginosa PAO1 (Cai et al., 1998), and an ArsH protein that is 63\% identical to ArsH from R64 (Sampei et al., 2010). Hence, all the genes required to confer resistance to arsenite and arsenate are present (Kaur et al., 2011). However, the E. coli transconjugants containing pIP40a did not grow on LB agar supplemented with $5 \mathrm{mM}$ sodium arsenite or $20 \mathrm{mM}$ sodium arsenate. This susceptibility to arsenate and arsenite indicates that there may be a defect in the operon or that the ArsR regulator of the operon may be inactive, and this will require further investigation.

GIsul2 also includes three genes encoding proteins that may be important for the stable maintenance of the genomic island (Fig. 4a), including a putative type 1 toxin (Pfam 12,703), the putative anti-toxin (PemI) sharing $42 \%$ amino acid identity with GenPept EXI82236, and a protein that belongs to the StbC superfamily of proteins (NCBI Conserved Domain cl01921) that is usually associated with plasmid stability.

A small 237 bp orf was found between the genes for the conjugative transfer proteins TrbJ (GenPept AOE47832) and TrbL (GenPept AOX98907) described previously. The predicted 78 aa protein (GenPept AOX98906) was identified as a putative entry exclusion lipoprotein TrbK (Fig. 4a), as it shares 33\% amino acid identity with the TrbK entry exclusion protein of the IncP1- $\alpha$ plasmid RP4 (RP1, RK2, R18, R68). TrbJ and TrbL of GIsul2 share $58 \%$ and $52 \%$ amino acid identity with TrbJ and TrbL from RP4, respectively. RP4 TrbL is believed to be involved in mating pair stabilization, whilst TrbJ is an inner membrane pore-forming protein (Pansegrau et al., 1994). TrbK in RP4 prevents other IncP1- $\alpha$ plasmids from using conjugation to enter the same cell (Haase et al., 1996). Due to the similarity between the Trb proteins encoded by genes on GIsul2 and IncP1- $\alpha$ plasmids, it is possible that GIsul2 interacts with IncP1- $\alpha$ or related plasmids. The similarity between the IncP1- $\alpha$ trbJ, trbK and trbL genes and the genes now known to belong to GIsul2 had been noted previously (Chiu and Thomas, 2004). However, at the time, the context of the genes had not been examined and GIsul2 had not yet been discovered.

\subsection{Stability of GIsul2 in pIP40a}

The int gene of GIsul2 encodes an Int (integrase, Pfam PF00589) protein that shares $89 \%$ amino acid identity with the SlpA integrase of the CP4-57 phage (Kirby et al., 1994). Interestingly, GIsul2 also includes a gene, alpA, encoding an AlpA transcriptional regulator (Pfam PF05930, GenPept AOX98904), which shares $84 \%$ aa identity with the AlpA (MerR-family) DNA binding protein of the CP4-57 prophage (Trempy et al., 1994). In the CP4-57 phage, AlpA positively regulates the SlpA integrase (Trempy et al., 1994), and increased SlpA leads to excision and loss of the prophage (Kirby et al., 1994). Hence, it is likely that Int of GIsul2 is regulated by AlpA in the same manner. Related Int and AlpA proteins have been found in a group of integrative elements that are found at the end of the guaA guanine monophosphate (GMP) synthase gene (Song et al., 2012).

As GIsul2 is an integrative element, under appropriate conditions it should be able to excise from the plasmid or chromosome in which it resides. To examine the stability of GIsul2 $\left(\mathrm{Su}^{\mathrm{R}}\right)$ in pIP40a, E. coli cells containing pIP40a were grown overnight ( $\sim 22$ generations) without sulphonamide selection. No sulphonamide-susceptible cells were detected among 600 colonies screened (200 colonies from each of three independent experiments), indicating that all cells tested had retained GIsul2. To test for low-frequency excision of the GI, whole cell DNA extracted after a single cycle of growth without antibiotic selection of cells containing pIP40a was used as a template in a PCR using primers RH1879 and R3R that face outwards from the int (RH1879) and sul2 (R3R) ends of GIsul2, and would be expected to generate an amplicon of $788 \mathrm{bp}$ if the excised circular form of GIsul2 (or a tandem duplication) is present. No amplicon was produced from whole cell DNA from three independent cultures of cells containing pIP40a, and re-amplification using the same primers also failed to produce an amplicon. Hence, GIsul2 is stable in pIP40a and specific conditions may be required to 
a

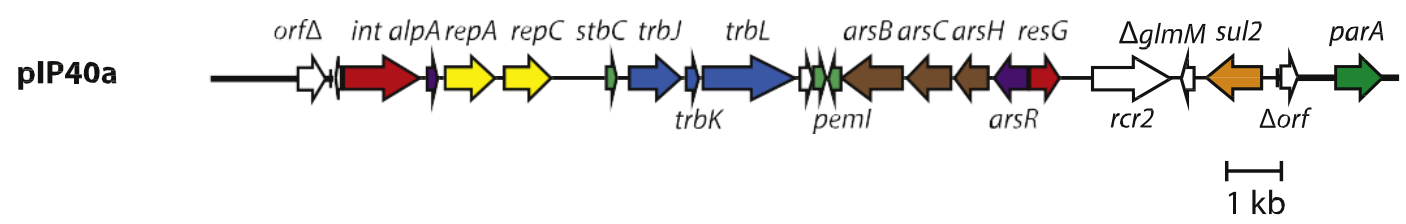

b

pIP40a (int end)

pRMH760

pIP40a (sul2 end)

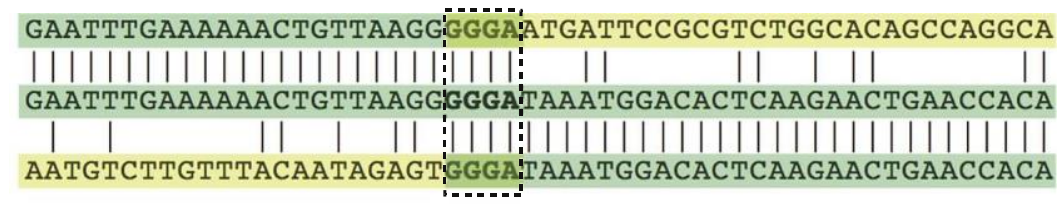

C

Type 1 (int end)

AAGAATTTGAAAAAACTGTTAAGGGGAGGA:ATGATCCGCTCTGGCACAGC

Type 2 (int end) ||||||||||||||||| |||||||||||||⿱|||||||||||||||||||||||||||||||||||||| AAGAATTTGAAAAAACAGTTAAGGGGGAGGGATMATTCCGCGTCTGGCACAGC

Type 1 (sul2 end)

Type 2 (sul2 end)

AATGTCTTGTTTACAATAGAGT:GG TAAATGGACACTCAAGAACTGAACCACA ||||||||||||||||||||||||||||||||||||||| ||||||||||||||| AATGTCTTGTTACAATAGAGT'GGGATAAATGGACGCTAAAGAACTGAACCACA

d

Glsul2

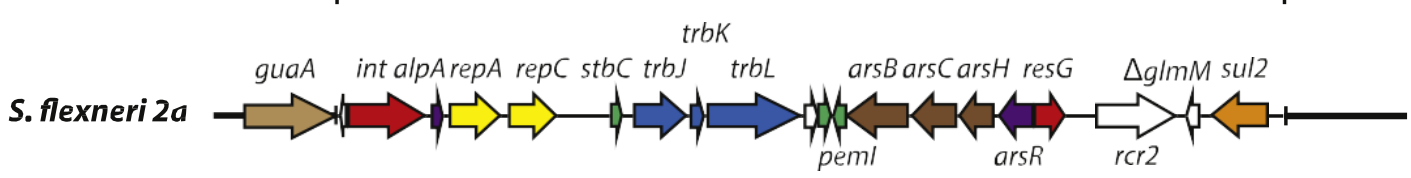

e
S. flexneri 2 a (int end)
AAGCCGCCAGCTACCATTGAGTGGGATGATTCCGCTCTGGCACAGCCAGGCACCTG
S. flexneri NCTC1 (guaA)
AAGCCGCCAGCTACCATTiGAGTGGGATGATTTTGACCCTGCACTATGAATGAACAA
S. flexneri 2 a (sul2 end)
||
|||| ||||||||\|\|\|\|\|\|\|\|\|\|\|\|\|\|\|\|\|\|\|\|
AATGTCTTGTTTACAATA'GAGTGGGATGATTTGATCCCTGCACTATGAATGAACAA

\section{S. flexneri chromosome}

Glsul2 backbone

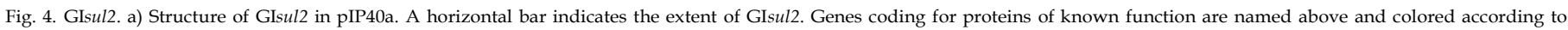

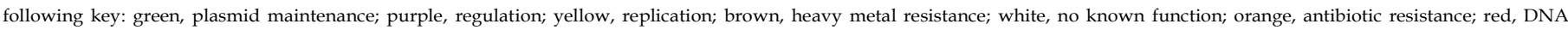

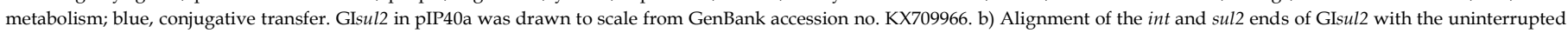

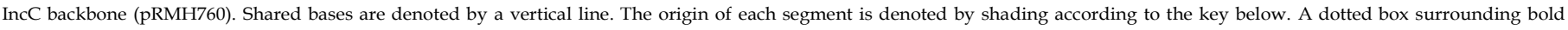

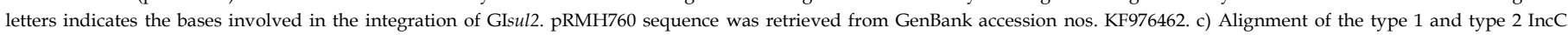

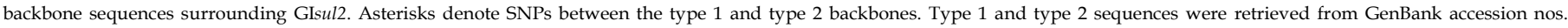

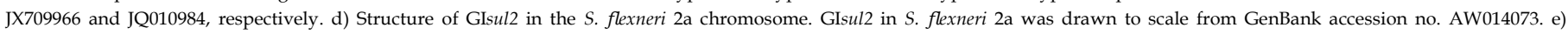

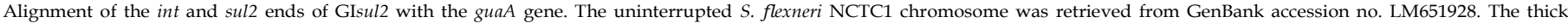

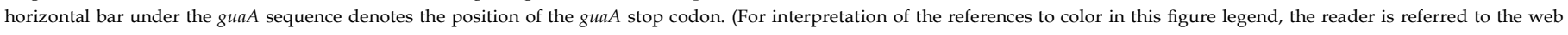
version of this article.)

induce the excision of GIsul2.

3.7. GIsul2 was acquired independently by type 1 and type 2 IncC plasmids

We have previously defined the four bases (GGGA) that are found at both the left and right boundaries of GIsul2 in the hybrid IncC plasmid p39R861-4 (Anantham et al., 2015), and the same bases are present at each end of GIsul2 in pIP40a (Fig. 4b). Only one GGGA is derived from the IncC backbone and the second is from GIsul2. The backbones of type 1 and type 2 IncC plasmids differ by approximately $1 \mathrm{bp} / 100 \mathrm{bp}$ 
Table 3

Features of GIsul2.

\begin{tabular}{|c|c|c|c|c|c|c|c|}
\hline Feature/Gene & Position $^{\mathrm{a}}$ & Size (bp) & Product & Size $(a a)^{b}$ & GenPept $^{c}$ & Pfam & Description \\
\hline orf $^{\mathrm{d}}$ & $43-219$ & 177 & orf & 58 & AOX98903 & - & Hypothetical protein \\
\hline int & $210-1508$ & 1302 & Int & 433 & AOE47829 & 00589 & Integrase \\
\hline $\operatorname{alp} A$ & $1659-1880$ & 222 & AlpA & 73 & AOX98904 & 05930 & Predicted DNA-binding transcriptional regulator \\
\hline repA & 1885-2712 & 828 & RepA & 275 & AOE47830 & 13481 & Replication initiation protein \\
\hline repC & $2843-3580$ & 738 & RepC & 245 & AOE47831 & 06504 & Replication initiation protein \\
\hline$s t b C$ & $4613-4801$ & 189 & StbC & 62 & AOX98905 & - & Plasmid stability protein \\
\hline $\operatorname{trbJ}$ & $4870-5718$ & 852 & TrbJ & 283 & AOE47832 & 07996 & Conjugative transfer protein \\
\hline $\operatorname{trbK}$ & $5734-5970$ & 237 & TrbK & 78 & AOX98906 & 10907 & Entry exclusion protein \\
\hline $\operatorname{trbL}$ & $5981-7408$ & 1428 & TrbL & 475 & AOX98907 & 04610 & Conjugative transfer protein \\
\hline orf & $7413-7661$ & 249 & orf & 82 & AOX98908 & - & Hypothetical protein \\
\hline orf & $7725-7949$ & 225 & orf & 74 & AOX98909 & 12703 & Putative toxin of toxin-antitoxin type 1 system \\
\hline pemI & $7986-8189 c$ & 204 & PemI & 67 & AOX98910 & - & Putative programmed cell death antitotin \\
\hline $\operatorname{ars} B$ & 8304-9371c & 1068 & ArsB & 355 & AOE47834 & 01758 & Arsenate/arsenite transporter protein \\
\hline $\operatorname{arsC}$ & $9368-9874 c$ & 507 & ArsC & 168 & AOE47835 & 01451 & Arsenate/arsenite reductase protein \\
\hline $\operatorname{arsH}$ & $9871-10638 \mathrm{c}$ & 768 & $\mathrm{ArsH}$ & 255 & AOE47836 & 03358 & Arsenate/arsenite resistance protein \\
\hline $\operatorname{ars} R$ & $10,635-10967 \mathrm{c}$ & 333 & ArsR & 110 & AOE47837 & 12840 & Arsenical resistance operon repressor \\
\hline resG & $11,115-11,852$ & 738 & ResG & 245 & AOE47838 & 02796 & Resolvase \\
\hline CR2 & $12,166-14,012$ & 1847 & - & - & - & - & Mobile element CR2 \\
\hline rcr 2 & $12,284-13,777$ & 1494 & Rcr2 & 497 & AOE47839 & 14319 & Mobilization protein \\
\hline sul2 & $14,342-15157 c$ & 816 & Sul2 & 271 & AOE47841 & 00809 & Confers resistance to sulfonamide antibiotics \\
\hline
\end{tabular}

a Position 1 corresponds to position 29940 of GenBank accession number KX709966. The letter "c" after the base range indicates the gene/orf is on the complimentary strand.

bength of product in amino acids (aa).

${ }^{c}$ GenPept from GenBank accession number JX709966.

${ }^{\mathrm{d}}$ Open reading frame.

(Harmer and Hall, 2014), and the presence of GIsul2 or a GIsul2 remnant in the same position and orientation in both type 1 and type 2 plasmids suggested that this location may be a site recognised by the GIsul2 Int. Examination of $1 \mathrm{~kb}$ of plasmid backbone sequence on either side of GIsul2 in pIP40a (type 1) and the GIsul2 remnant in R55 (type 2) revealed that it corresponds to the type 1 and type 2 backbones, respectively. In fact, the closest single basepair differences are 8 away from the conserved GGGA on one side and $10 \mathrm{bp}$ away on the other (Fig.4c). If GIsul2 had been acquired once then spread via homologous recombination the crossovers would have been within these 8 and 10 bp spans. Hence, this element has been independently acquired by the two lineages.

\subsection{Distribution of GIsul2}

We previously reported that complete copies of GIsul2 were found in bacterial chromosomes of four species and in two plasmids (Anantham et al., 2015; Nigro and Hall, 2011). In the chromosomes, GIsul2 was located in the end of the core guaA GMP synthetase gene (Anantham et al., 2015; Nigro and Hall, 2011). An updated search for sequences in the GenBank non-redundant database (October 2016), and a recent report of a complete GIsul2 in the draft genome of Acinetobacter baumannii ATCC 19606 (Hamidian and Hall, 2017), revealed that complete copies of GIsul2 are now found in a total of 11 chromosomes from a variety of Gram negative bacterial species (Table 4). In nine out of the 11 chromosomes, GIsul2 is located in the end of the core guaA gene (Fig. 4d), always in the same orientation, and this is clearly the preferred target site. GIsul2 inserts $4 \mathrm{bp}$ from the 3 '-end of guaA within an 8-bp concensus sequence (GAGTGGGA, Fig. 4e), which was found at both the left and right boundaries of the element and this sequence was also noted for related elements that target this site (Song et al., 2012). The next four bases at the int end of GIsul2 (ATGA) restore the stop codon in guaA.

In A. baumannii strains 3207 (GenBank accession number CP015364) and ATCC19606 (Hamidian and Hall, 2017), the sequence surrounding GIsul2 is not normally part of the A. baumannii chromosome, and GIsul2 appears to be in a larger genomic island. Complete copies of GIsul2 were also found in five plasmid sequences in addition to pIP40a (Table 4). The plasmids belong to incompatibility groups IncB/O, and IncX. Two plasmids from Acinetobacter species are of an undetermined type. The same four bases are also conserved surrounding GIsul2 in the plasmids of different incompatibility types listed in Table 4, and these too may represent Int-specific sites.

\section{Discussion}

pIP40a is one of the oldest known IncC plasmids recovered to-date. However, pIP40a had already acquired a novel mercury resistance transposon, Tn6182, as well as Tn6023 (conferring kanamycin and neomycin resistance), a partial copy of $\operatorname{Tn} 3$ (conferring resistance to ampicillin), Tn1000, and GIsul2. pIP40a represents a lineage of type 1 IncC plasmids that is yet to acquire the ARI-A resistance island that plays such a key role in resistance gene accumulation, and pDGO100, from 1981 (Harmer et al., 2016b), is currently the earliest known example of a type 1 plasmid containing ARI-A. However, pDGO100 does not contain ARI-B and therefore cannot be directly descended from pIP40a. As GIsul2 has been introduced on at least two separate occasions into the same site in a type 1 and type 2 IncC plasmid, it may have been acquired by the type 1 lineage on more than one occasion. The ancestor of the type 1 lineage that contains neither the ARI-A nor the ARI-B islands is still to be discovered. The Murray Collection of preantibiotic era strains recovered as early as 1917 was recently sequenced (Baker et al., 2015). Though it may have been a source of ancestral IncC plasmids, a search of the sequencing metadata showed that no IncC plasmids were detected in the collection.

Subsequent to the release of our pIP40a sequence (Harmer and Hall, 2016), another pIP40a sequence was reported (Szabo et al., 2016) (GenBank accession number KX156772). Interestingly, whilst the sequences are very similar, there are some differences that are likely to have accumulated over time, during storage and passage of the plasmid in different laboratories. The second sequence lacks the IS3, present in the rhs1 gene of our sequence, and contains three additional IS not present in our version of pIP40a; an IS2 inserted in a gene encoding a hypothetical protein (orf 21 in Fig. 1), an IS186 in an intergenic region in the backbone, between orf106 and pri, and an IS150 in the int gene of GIsul2. The two backbones also differ by six single base pair differences. Comparison to other sequenced IncC plasmids showed that the six differences are unique to our $\mathrm{pIP} 40 \mathrm{a}$ sequence, with all other sequences sharing the same bases as GenBank accession number KX156772. One of these single base substitutions occurs in an intergenic region, and one 
Table 4

Examples of complete GIsul2 in sequenced strains.

\begin{tabular}{|c|c|c|c|c|c|}
\hline Organism & Host/Plasmid name & Year of isolation & GIsul2 location ${ }^{\mathrm{a}}$ & Insertions & GenBank accession \\
\hline S. flexneri & 2a str $2457 \mathrm{~T}$ & 1954 & C-guaA & - & AE014073 \\
\hline S. flexneri & G1663 & - & C-guaA & - & СР007037 \\
\hline P. stuartii & ATCC 33672 & - & C-guaA & - & CP008920 \\
\hline E. cloacae & ATCC 13047 & 1890 & C - guaA & $\operatorname{Tn} 5393$ & СР001918 \\
\hline S. granuli & TFA & 1995 & C- guaA & - & СР012199 \\
\hline M. morganii & FDAARGOS_172 & 2014 & C - guaA & - & СР014026 \\
\hline A. xylosoxidans & NCTC10807 & 1971 & C - guaA & - & LN831029 \\
\hline E. coli & 6409 & 2012 & C - guaA & - & СР010371 \\
\hline Pseudomonadaceae spp & E5571 & 1979 & C - guaA & - & CP012365 \\
\hline A. baumannii & 3207 & 2008 & $r^{b}$ & - & СР015364 \\
\hline A. baumannii & ATCC19606 & 1948 & $C^{c}$ & - & $-{ }^{\mathrm{d}}$ \\
\hline P. aeruginosa & pIP40a & 1969 & $\mathrm{P}$ (IncC) & - & KX709966 ${ }^{\mathrm{e}}$ \\
\hline E. coli & R16a & 1966 & $\mathrm{P}($ IncC) & - & KX156773 \\
\hline E. coli & pHUSEC411-like & - & $\mathrm{P}(\mathrm{IncB} / \mathrm{O})$ & - & HG428756 \\
\hline A. calcoaceticus & NCTC7364 & 2014 & $\mathrm{P}$ & ISAba1 & LT605060 \\
\hline A. baumannii & ATCC17978-mff & 1951 & $\mathrm{P}$ & ISAba1 & СР012005 \\
\hline M. morganii & R485 & 1972 & $P(\operatorname{Inc} X)$ & - & HE577112 \\
\hline
\end{tabular}

${ }^{\text {a }} \mathrm{C}$ - guaA denotes insertion into the end of the guaA gene in the chromosome. P denotes plasmid. Name in brackets indicates plasmid incompatibility group, if known.

${ }^{\mathrm{b}}$ Sequence surrounding GIsul2 is not guaA and is unique to this strain.

${ }^{c}$ GIsul2 is located within a larger genomic island in the chromosome.

${ }^{\mathrm{d}}$ Draft genome. See Hamidian and Hall, 2017.

${ }^{\mathrm{e}}$ See also GenBank accession number KX156772.

is a missing base pair in pri that introduces a frame shift, extending the 576 aa Pri to a 586 aa protein. Two other single base substitutions are silent mutations in $s t b A$ and orf1832. The remaining two base pair differences result in the leucine usually found at position 113 of Int being replaced with an arginine, and the lysine at position 293 of Dcm3 being replaced with an arginine.

The same study also reported the sequence of R16a (Szabo et al., 2016), recovered three years earlier in 1966, from the St-Antoine Hospital in Paris (Chabbert et al., 1972). This type 1 IncC plasmid also includes a complete copy of GIsul2 and does not contain an ARI-A island. It carries Tn6182 in the same position as seen in pIP40a, but the Tn6182 is interrupted by additional transposons that have introduced genes conferring resistance to ampicillin (Tn1, bla $\left.a_{\mathrm{TEM}-1}\right)$, and kanamycin and neomycin (Tn6020, aphA1b). Hence, Tn6020, which was first detected in 2009 (Post and Hall, 2009) had been circulating much earlier, likely in response to the use of kanamycin, as had Tn6023 seen in pIP40a. These transposons continue to be found in modern day plasmids of various types. In contrast, this is the only report of Tn6182.

The analysis of the pIP40a sequence also uncovered previously unnoticed functions encoded by genes in the genomic island GIsul2. An apparently complete arsenate and arsenite resistance operon does not appear to confer resistance to these heavy metals. The similarity between the Int protein of GIsul2 and the SlpA integrase of the CP4-57 phage, which is known to be active (Kirby et al., 1994), indicates the role that int, and the adjacent alpA, are likely to play in the biology of GIsul2. However, we were unable to detect excision and further experiments will be required to confirm the role of AlpA in the regulation of Int, and the subsequent Int-mediated excision of GIsul2.

The significant levels of amino acid identity between the TrbJ, TrbK and TrbL proteins of GIsul2 and those found in IncP plasmids suggests that GIsul2 may be mobilized by IncP plasmids in much the same way that IncA and IncC plasmids are able to mobilize SGI1 and SGI2 type Salmonella genomic islands (Hall, 2010; Harmer et al., 2016a; Douard et al., 2010; Doublet et al., 2005; Kiss et al., 2015; Carraro et al., 2014a). This warrants further investigation. GIsul2 also includes three genes encoding toxin-antitoxin proteins and a stability protein, which may be involved in the stable maintenance of the island once it has integrated into the chromosome or a plasmid.

\section{Abbreviations}

$\begin{array}{ll}\text { GI } & \text { genomic island } \\ \text { IR } & \text { inverted repeats } \\ \text { IS } & \text { insertion sequence } \\ \text { ORF } & \text { open reading frame } \\ \text { SNP } & \text { single nucleotide polymorphism } \\ \text { TSD } & \text { target site duplications }\end{array}$

Funding information

This work and CJH were supported by NHMRC grant 1086267. MH was supported by NHMRC grant 1079616.

\section{References}

Anantham, S., Harmer, C.J., Hall, R.M., 2015. p39R861-4, a type 2 A/C plasmid carrying a segment from the A/C plasmid RA1. Microb. Drug Resist. 21, 571-576.

Bailey, J.K., Pinyon, J.L., Anantham, S., Hall, R.M., 2010. Commensal Escherichia coli of healthy humans: a reservoir for antibiotic-resistance determinants. J. Med. Microbiol. 59, $1331-1339$.

Bailey, J.K., Pinyon, J.L., Anantham, S., Hall, R.M., 2011. Distribution of the blaTEM gene and blaTEM-containing transposons in commensal Escherichia coli. J. Antimicrob. Chemother. 66, 745-751.

Baker, K.S., Burnett, E., Mcgregor, H., Deheer-Graham, A., Boinett, C., Langridge, G.C., Wailan, A.M., Cain, A.K., Thomson, N.R., Russell, J.E., Parkhill, J., 2015. The Murray collection of pre-antibiotic era Enterobacteriacae: a unique research resource. Genome Med. 7, 97.

Broom, J.E., Hill, D.F., Hughes, G., Jones, W.A., Mcnaughton, J.C., Stockwell, P.A., Petersen, G.B., 1995. Sequence of a transposon identified as Tn1000 (gamma delta). DNA Seq. 5, 185-189.

Cai, J., Salmon, K., Dubow, M.S., 1998. A chromosomal ars operon homologue of Pseudomonas aeruginosa confers increased resistance to arsenic and antimony in Escherichia coli. Microbiology 144, 2705-2713.

Cain, A.K., Hall, R.M., 2011. Transposon Tn5393e carrying the aphA1-containing transposon Tn6023 upstream of strAB does not confer resistance to streptomycin. Microb. Drug Resist. 17, 389-394.

Carattoli, A., Miriagou, V., Bertini, A., Loli, A., Colinon, C., Villa, L., Whichard, J.M., Rossolini, G.M., 2006. Replicon typing of plasmids encoding resistance to newer betalactams. Emerg. Infect. Dis. 12, 1145-1148.

Carraro, N., Matteau, C., Luo, P., Rodrigue, S., Burrus, V., 2014a. The master activator of Inc $\mathrm{A} / \mathrm{C}$ conjugative plasmids stimulates genomic islands and multidrug resistance dissemination. PLoS Genet. 10, e1004714.

Carraro, N., Sauve, M., Matteau, D., Lauzon, G., Rodrigue, S., Burrus, V., 2014b. Development of pVCR94DeltaX from Vibrio cholerae, a prototype for studying multidrug resistant IncA/C conjugative plasmids. Front. Microbiol. 5, 44.

Chabbert, Y.A., Scavizzi, M.R., Witchitz, J.L., Gerbaud, G.R., Bouanchaud, D.H., 1972. Incompatibility groups and the classification of $f i$-resistance factors. J. Bacteriol. $112,666-675$. 
Chiu, C.M., Thomas, C.M., 2004. Evidence for past integration of IncP-1 plasmids into bacterial chromosomes. FEMS Microbiol. Lett. 241, 163-169.

Datta, N., Hedges, R.W., 1972. R factors identified in Paris, some conferring gentamicin resistance, constitute a new compatibility group. Ann. Inst. Pasteur (Paris) 123, 849-852.

Datta, N., Hedges, R.W., 1973. R factors of compatibility group A. J. Gen. Microbiol. 74 335-337.

Douard, G., Praud, K., Cloeckaert, A., Doublet, B., 2010. The Salmonella genomic island 1 is specifically mobilized in trans by the IncA/C multidrug resistance plasmid family. PLoS One 5, e15302.

Doublet, B., Boyd, D., Mulvey, M.R., Cloeckaert, A., 2005. The Salmonella genomic island 1 is an integrative mobilizable element. Mol. Microbiol. 55, 1911-1924.

Doublet, B., Boyd, D., Douard, G., Praud, K., Cloeckaert, A., Mulvey, M.R., 2012. Complete nucleotide sequence of the multidrug resistance Inc $\mathrm{A} / \mathrm{C}$ plasmid pR55 from Klebsiella pneumoniae isolated in 1969. J. Antimicrob. Chemother. 67, 2354-2360.

Fernandez-Alarcon, C., Singer, R.S., Johnson, T.J., 2011. Comparative genomics of multidrug resistance-encoding IncA/C plasmids from commensal and pathogenic Escherichia coli from multiple animal sources. PLoS One 6, e23415.

Groot Obbink, D.J., Ritchie, L.J., Cameron, F.H., Mattick, J.S., Ackerman, V.P., 1985. Construction of a gentamicin resistance gene probe for epidemiological studies. Antimicrob. Agents Chemother. 28, 96-102.

Haase, J., Kalkum, M., Lanka, E., 1996. TrbK, a small cytoplasmic membrane lipoprotein, functions in entry exclusion of the IncP alpha plasmid RP4. J. Bacteriol. 178, 67206729.

Hall, R.M., 2010. Salmonella genomic islands and antibiotic resistance in Salmonella enterica. Future Microbiol 5, 1525-1538.

Hamidian, M., Hall, R.M., 2017. Acinetobacter baumannii ATCC 19606 carries GIsul2 in a genomic island located in the chromosome. Antimicrob. Agents Chemother. 61 (e01991-16).

Hancock, S.J., Phan, M.D., Peters, K.M., Forde, B.M., Chong, T.M., Yin, W.F., Chan, K.G., Paterson, D.L., Walsh, T.R., Beatson, S.A., Schembri, M.A., 2017. Identification of IncA/C plasmid replication and maintenance genes and development of a plasmid Multilocus sequence typing scheme. Antimicrob. Agents Chemother.. http://dx.doi. org/10.1128/AAC.01740-16. (In press).

Harmer, C.J., Hall, R.M., 2014. pRMH760, a precursor of A/C2 plasmids carrying bla $a_{\mathrm{CM}}$ and bla $a_{\text {NDM }}$ genes. Microb. Drug Resist. 20, 416-423.

Harmer, C.J., Hall, R.M., 2015. The A to Z of A/C plasmids. Plasmid 80, 63-82.

Harmer, C.J., Hall, R.M., 2016. PCR-based typing of IncC plasmids. Plasmid 87-88, 37-42.

Harmer, C.J., Hamidian, M., Ambrose, S.J., Hall, R.M., 2016a. Destabilization of IncA and IncC plasmids by SGI1 and SGI2 type Salmonella genomic islands. Plasmid 87-88, $51-57$.

Harmer, C.J., Partridge, S.R., Hall, R.M., 2016b. pDGO100, a type 1 IncC plasmid from 1981 carrying ARI-A and a Tn1696-like transposon in a novel integrating element. Plasmid 86, 38-45.

Kaur, S., Kamli, M.R., Ali, A., 2011. Role of arsenic and its resistance in nature. Can. J.
Microbiol. 57, 769-774.

Kirby, J.E., Trempy, J.E., Gottesman, S., 1994. Excision of a P4-like cryptic prophage leads to Alp protease expression in Escherichia coli. J. Bacteriol. 176, 2068-2081.

Kiss, J., Papp, P.P., Szabo, M., Farkas, T., Muranyi, G., Szakallas, E., Olasz, F., 2015. The master regulator of IncA/C plasmids is recognized by the Salmonella genomic island SGI1 as a signal for excision and conjugal transfer. Nucleic Acids Res. 43, 8735-8745.

Liebert, C.A., Hall, R.M., Summers, A.O., 1999. Transposon Tn21, flagship of the floating genome. Microbiol. Mol. Biol. Rev. 63, 507-522.

Nicolas, E., Lambin, M., Dandoy, D., Galloy, C., Nguyen, N., Oger, C.A., Hallet, B., 2015. The Tn3-family of replicative transposons. Microbiol Spectr. 3.

Nigro, S.J., Hall, R.M., 2011. GIsul2, a genomic island carrying the sul2 sulphonamide resistance gene and the small mobile element CR2 found in the Enterobacter cloacae subspecies cloacae type strain ATCC 13047 from 1890, Shigella flexneri ATCC 700930 from 1954 and Acinetobacter baumannii ATCC 17978 from 1951. J. Antimicrob. Chemother. 66, 2175-2176.

Pansegrau, W., Lanka, E., Barth, P.T., Figurski, D.H., Guiney, D.G., Haas, D., Helinski, D.R.,Schwab, H., Stanisich, V.A., Thomas, C.M., 1994. Completenucleotidesequence of Birmingham IncP alpha plasmids. Compilation and comparative analysis. J. Mol. Biol. 239, 623-663.

Partridge, S.R., Hall, R.M., 2005. Evolution of transposons containing blaTEM genes Antimicrob. Agents Chemother. 49, 1267-1268.

Partridge, S.R., Brown, H.J., Stokes, H.W., Hall, R.M., 2001. Transposons Tn1696 and Tn21 and their integrons In 4 and In2 have independent origins. Antimicrob. Agents Chemother. 45, 1263-1270.

Post, V., Hall, R.M., 2009. AbaR5, a large multiple-antibiotic resistance region found in Acinetobacter baumannii. Antimicrob. Agents Chemother. 53, 2667-2671.

Sampei, G., Furuya, N., Tachibana, K., Saitou, Y., Suzuki, T., Mizobuchi, K., Komano, T., 2010. Complete genome sequence of the incompatibility group I1 plasmid R64. Plasmid 64, 92-103.

Siguier, P., Perochon, J., Lestrade, L., Mahillon, J., Chandler, M., 2006. ISfinder: the reference centre for bacterial insertion sequences. Nucleic Acids Res. 34, D32-D36.

Song, L., Pan, Y., Chen, S., Zhang, X., 2012. Structural characteristics of genomic islands associated with GMP synthases as integration hotspot among sequenced microbial genomes. Comput. Biol. Chem. 36, 62-70.

Szabo, M., Nagy, T., Wilk, T., Farkas, T., Hegyi, A., Olasz, F., Kiss, J., 2016. Characterization of Two Multidrug-resistant IncA/C Plasmids from the 1960s by Using Oxford Nanopore MinION Sequencer Device. (Antimicrob Agents Chemother).

Trempy, J.E., Kirby, J.E., Gottesman, S., 1994. Alp suppression of Lon: dependence on the slpA gene. J. Bacteriol. 176, 2061-2067.

Welch, T.J., Fricke, W.F., Mcdermott, P.F., White, D.G., Rosso, M.L., Rasko, D.A., Mammel, M.K., Eppinger, M., Rosovitz, M.J., Wagner, D., Rahalison, L., Leclerc, J.E., Hinshaw, J.M., Lindler, L.E., Cebula, T.A., Carniel, E., Ravel, J., 2007. Multiple antimicrobial resistance in plague: an emerging public health risk. PLoS One 2, e309.

Witchitz, J.L., Chabbert, Y.A., 1971. High level transferable resistance to gentamicin. J. Antibiot. (Tokyo) 24, 137-139. 